\title{
3. The reality of information systems research
}

\author{
John Lamp, School of Information Systems, Deakin Univer- \\ sity
}

Simon Milton, Department of Information Systems, University of Melbourne

\begin{abstract}
The examination of a practical issue with a web site has led, in this paper, directly to the consideration of the need for, and an assessment of the impact of, an approach based on fundamental theories of 'what is', to examine what information systems research is and the relations of its component areas of endeavour. The paper presents an examination of the use of the philosophical field of ontologies, and specifically the use of the ontological approaches upon which to base categories of information systems research activities. This theoretical analysis is intended to be used as the basis from which to develop a methodology to undertake the development of the categorial scheme for the web site that initiated the research.
\end{abstract}

\section{Introduction}

Since 1995, one of the authors (JL) has been maintaining a resource on the World Wide Web with the basic aim of providing a central point from which academic authors publishing in the information systems domain can obtain useful information on the publications serving that domain (Lamp, 1995). The database now contains information on 349 journals, and was accessed over 7500 times in February 2004. As the number of journals included in the database increases, so also does the difficulty of accurately identifying journals relevant to a particular query from within the database. There is a basic searching facility that simply matches a search term to descriptive entries in the database but, in common with most text searches implemented on a relational database management system (DBMS), there is no facility for maintaining result sets and refining searches through the manipulation of result sets (Ramakrishnan and Gehrke, 2003).

A number of users have asked whether it would be possible to categorise the journals according to their subject area. This has led to a research project, the full scope of which has been reported elsewhere (Lamp and Milton, 2003), but a key part of which is the determination of relevant categorisation schemes for information systems research, and the relations between those categories. It has also raised important questions about the nature of the discipline of information systems, the journals themselves, the articles published in them, and the readers of those journals. A key finding of Lamp and Milton (2003) was the lack of widespread adoption of any categorial scheme over the domain of information systems research. This is in apparent contradiction with views expressed by information systems researchers, as researchers and as journal editors (Lamp, 2002), supporting the need for such a scheme. 
We assert that the artefacts of research (i.e. journal articles and other publications) are real, having an existence outside the cognition of their authors and readers. The question then arises as to how we can categorise these objects.

The method of this paper is as follows:

1. First, we establish that the information systems research domain is diverse.

2. Second, we examine the applicability of philosophical ontology as a tool to explore the diverse categorisation of the reality of information systems research and its community; in particular, the nature of the real artefacts, how people relate to that reality, and to identify a philosophy upon which to build methods for analysis.

\section{Characterising information systems research}

There are a number of publications dealing with what information systems are, and what information systems research is. From these papers, a number of common findings emerge:

1. There is often debate on what information systems is (Ives, et al., 1980; Seddon, 1991; Shanks, et al., 1993; Parker, et al., 1994; Holsapple, et al., 1994);

2. Information systems (IS) has many foundation or reference disciplines (Keen, 1991; Seddon, 1991; Avison, 1993; Holsapple, et al., 1994; Parker, et al., 1994; Walczak, 1999; Galliers, 2004);

3. IS is located in different university faculties (Avison, 1993; Holsapple, et al., 1994);

4. IS is perceived as weak on theory (Keen, 1991; Avison, 1993; Straub, et al., 1994; Gregor, 2002);

5. IS is perceived as practice dominated (Hurt, et al., 1986; Keen, 1991; Avison, 1993, Shanks, et al., 1993);

6. IS uses many different research methodologies, models or frameworks (Ives, et al., 1980; Avison, 1993, Shanks, et al., 1993; Holsapple, et al., 1994; Parker, et al., 1994; Straub, et al., 1994; Baskerville and Wood-Harper, 1998; Fitzgerald and Howcroft, 1998; Galliers, 2004).

The authors clearly perceive that both the nature and scope of the information systems domain are diverse; the approaches to researching information systems are diverse; the approaches to teaching information systems are diverse and that there is a lack of any single clear theoretical basis for the study of information systems.

The information systems research literature is characterised by only token adoption of any form of subject categorisation, whether proposed as specific to the information systems discipline or imported, with or without adoption, from one of the reference disciplines (Lamp and Milton, 2003). The degree to which an information system is adopted by users has long been used as a determinant of success (DeLone and McLean, 1992) and, in like manner, the lack of adoption of existing categorisation schemes may be seen as an indication of the failure of those subject categorisation schemes.

All of the subject categorisation schemes that have been applied to the information systems domain have had simple hierarchical structures, enforcing a single view of information systems subject categorisation. It could be hypothesised that such a single structured subject categorisation scheme is inadequate to capture the diversity inherent in the information systems domain, resulting in the lack of significant uptake of any of these schemes.

How then can this diversity be expressed in a categorisation scheme? Systems theorists (Ackoff and Emery, 1972; Checkland, 1981; Churchman, 1979) have identified the need to account for diversity, whether expressed as perspectives or as world views, in the 
models they create. While these approaches reflect the diversity of perspectives in information systems, they deal with different units of analysis. Rather than examining a possibly hypothetical system and the processes and entities that exist in that system, we are examining things that exist in reality, and such a study has its roots in ontology.

\section{Ontology}

Within philosophy, ontology is the study of things that exist; the objects, properties, categories and relations that make up the world. A definition of ontology as it is conceived by philosophers is:

... the science of being in general, embracing such issues as the nature of existence and the categorial structure of reality.... Different systems of ontology propose alternative categorial schemes. A categorial scheme typically exhibits a hierarchical structure, with 'being' or 'entity' as the topmost category, embracing everything that exists (Honderich, 1995).

This approach to the definition and use of ontology has also been successfully applied in information systems, for example in comparing and evaluating data modelling frameworks (Milton, 2000; Milton and Kazmierczak, 2004).

Philosophers distinguish between reference ontologies, which aim to determine the fundamental categories and categorial structures, and domain specific ontologies, where a particular reference ontology is applied to a certain problem domain. An analogy could be made between the way that ontologies and data models are conceptualised, as shown in Table 3.1.

\section{Table 3.1. Ontologies and data models}

\begin{tabular}{|l|l|}
\hline Ontology & Data model \\
\hline Reference ontology & Data modelling language \\
\hline Domain specific ontologies & Data models \\
\hline Specific reality & $\begin{array}{l}\text { Database implementation of specific database } \\
\text { instances }\end{array}$ \\
\hline
\end{tabular}

\section{Reference ontologies}

Reference ontologies, such as that of Bunge (1977; 1979), Chisholm (1996), Basic Formal Ontology (Smith, 1978; Smith and Mulligan, 1983) and DOLCE (Gangemi, et al., 2002) are concerned with the most general categories of what there is in the world. They deal with concepts such as thing, individual and property. In the analogy proposed in Table 3.1, data modelling languages such as entity-relationship modelling are proposed as analogous to reference ontologies.

\section{Domain specific ontologies}

At this level, particular reference ontologies are used to create a domain specific ontology directed towards an aspect of reality, in the way that particular data modelling languages are used to create data models of a particular project, such as a university student records system or a banking system. Early artificial intelligence (AI) focus was at this level.

\section{Ontology and artificial intelligence}

At this point we note that AI researchers have a particular view of ontology, referring to it as

... an engineering artefact, constituted by a specific vocabulary used to describe a certain reality, plus a set of explicit assumptions regarding the intended meaning of the vocabulary words (Guarino, 1998). 
As previously mentioned, ontologies developed within the AI community are often directed to a specific domain of knowledge in a specific context, and are intended to be implemented or defined within a specific software artefact. Examples are KIF (Genesereth and Fikes, 1992), Ontolingua (Gruber, 1992; 1995), and OIL (Fensel, et al., 2000).

Recent work by AI researchers around the Semantic Web and the IEEE Standard Upper Ontology (IEEE, 2003), and DOLCE (Gangemi, et al., 2002) is encouraging in that they all recognise the central role of higher level ontologies in information systems.

It is the higher level reference ontologies and the basis for the selection of a particular reference ontology for the creation of a domain specific ontology, directed towards information systems research, that is the concern of this paper.

\section{Approaches to categorisation}

The history of ontology goes back over 2000 years to Aristotle and his Categories. The influence of his concept of hierarchical structures can be seen in many current categorisation schemes, ranging from the Linnaean schemes used in the life sciences to the various library categorisation schemes of Dewey and the Library of Congress. The history of the study of ontology has not been one of smooth progress with, for example, Kant (1787, p. 80) even rejecting ontology as a valid area of study. Kant's attitude was based on his belief that ontology was a synthetic a priori knowledge of things in general. Franz Brentano (1933, p. 81) asserts that Kant completely misunderstood Aristotle's theory of categories, approaching it without a sound understanding of Aristotle's point of view. Rather than an a priori assumption, as Kant believed, Aristotle's categories were based on empirical observations of being, of individuation and of collection into species.

Despite Brentano's rejection of Kant, some of his views regarding perception had similarities. Brentano's ontology is based on the investigation of two types of phenomena. First, he identifies our inner perceptions, facts about ourselves and our intentional activity. Second, the sensations we have of the external world: colour, sounds, smells are identified. In his comments on intentionality we begin to see a way of accommodating perceived diversity:

However various our ideas of things may be, the differences of the ideas are not just a function of the differences of the objects of thought; they are also a function of what the object is thought of as. It is possible that the objects may differ and yet that one and the same idea may serve to present either one of them. And also conversely: one and the same object may be presented by two ideas which differ with respect to the object (Brentano, 1933, p. 40 [emphasis in original])

One of Brentano's students was Edmund Husserl, who continued investigations into the concept of intentionality. In the course of this he created phenomenology, the key idea of which is intentionality. Husserl identified problems with Brentano's approach when dealing with serious misperception and hallucinations. In these cases, what is the object? Husserl considered those features of consciousness that make it as if of an object. The collection of all these features he called the act's noema. The noema includes all the object's features, whether perceived or unperceived, including features we may take the object as having without having ever reflected or paid attention to those features. Because this is determined by perception you could, in a particular situation, see a man, but later realise it was a mannequin, with a corresponding shift of noema. An example closer to our research area might be Isaac Asimov's The endochronic properties of resublimated thiotimoline (1948). This is written using the structure and language of a chemistry re- 
search paper, but was a parody intended to entertain, and not a scientific work intended to establish and transmit cognitive results. Some members of the research community took the paper seriously until the ruse was revealed. In this way, perception is always fallible and noema must shift with reconsidered perceptions (Routledge, 2000, p. 369).

The results of information systems research are reported in the research literature. For this reason, and because the Index of Information Systems Journals can be considered a surrogate of information systems research literature when considering relevant search terms, examining and understanding what the research literature consists of is fundamental to understanding the domain of information systems research, and for discovering other dimensions of the literature which might be useful as search attributes. The philosophical approach reviewed thus far resulted in a seminal investigation of literary works.

The results of information systems research are reported in the research literature. For this reason, and because the Index of Information Systems Journals can be considered a surrogate of information systems research literature when considering relevant search terms, examining and understanding what the research literature consists of is fundamental to understanding the domain of information systems research, and for discovering other dimensions of the literature which might be useful as search attributes. The philosophical approach reviewed thus far resulted in a seminal investigation of literary works.

\section{Approaches to the literary work of art}

Roman Ingarden developed and applied Husslerian phenomenology to the examination of literary works, including scientific works as a borderline case. His two books The literary work of art (1965) and The cognition of the literary work of art (1968) provide a powerful framework of conceptual and methodological tools with which he characterised literary works. His work was comprehensive and addressed the entire range of literary works, from classic literature to, in his words, '... the serialised crime novel or a schoolboy's banal love poem' (Ingarden, 1965, p. 8). Fortunately for this study, he also explicitly included 'scientific works [which are] clearly distinguishable from the works of so-called belles-lettres .... and yet frequently spoken of as having greater or lesser literary value or as being devoid of it' (Ingarden, 1965, p. 9).

Ingarden proposed that literary works have a number of strata and that it is the characteristics of and diversity between strata that generate a polyphonic character to the work. These strata were described by him as:

1. the stratum of word sounds and the phonetic formations of higher order built upon them;

2. the stratum of meaning units of various orders;

3. the stratum of represented objectivities and their vicissitudes; and

4. the stratum of manifold schematised aspects and aspect continua and series.

In addition, he identified a fifth characteristic as being significant - the order of sequence of the literary work. Key in the examination of the various strata is identifying the connections between them. A detailed description of these strata and their various connections and contributions to the literary work of art is beyond the scope of this paper. It is important to note, however, that Ingarden saw these aspects as being applicable to scientific works. He proposed that scientific work differed in some elements of individual strata and the roles of the strata. The differences result from the role of scientific works 
in establishing the cognitive results attained and transmitting them to other conscious subjects. Ingarden (1965, pp. 329-30; 1968, pp. 146-53) identified five differences:

1. Sentences that appear in a scientific work are almost exclusively true judgments. Such sentences may be true or false, but they lay claim to truthfulness; for example, a paper may report 'The management style of company A was undemocratic', which is a result perceived as true by the author of the paper, and yet a second researcher may report a different result.

2. The structure of a scientific work naturally consists of purely intentional sentence correlates (almost exclusively states of affairs) and represented objectivities. This means that intentions are directed through the represented or portrayed objectivities on to objects independent of the scientific work (e.g. the real world).

3. Scientific works may, at the stratum of phonetic formations and the stratum of units of meaning, contain aesthetic value qualities. This is not essential and may be regarded as a dispensable luxury. The central purpose of a scientific work is cognitive exchange, and everything else must be subordinated to this central purpose. Ideally the portrayed objectivities are transparent to the reader and ontically independent objects are seen in the light of the meaning intention of the scientific work. For example, information technology is rich in accepted metaphor. The use of terms such as 'viruses', 'windows' and 'mice' in information technology literature assists in cognitive exchange.

4. Scientific works can contain, as a special stratum, manifolds of schematicised aspects held in readiness, provided the sentences refer to objects that can appear in manifolds of aspects. If they exist, their role is to assist in the transmission of cognitive results. The presence of decorative moments is dispensable and may be a hindrance.

5. The possible manifestation of metaphysical qualities is essential only when a given metaphysical quality is itself a subject of the cognitive result that is achieved and transmitted, or at least contributes to its transmission. In this case, they are not contributing to the aesthetic value of the scientific work in the way that they contribute to a literary work. Scientific literature tends to stick to facts.

From this discussion we can conclude three important things:

1. the journals and their articles are real (part of reality);

2. the articles contain true judgments of the authors; and

3. independent objects mentioned in articles are also real but must be understood from the perspective of the author and the intentions revealed in the work.

The approach taken by Ingarden is applicable to scientific works, including works in the field of information systems. This leaves the question of what is an appropriate reference ontology to provide a framework for linking the results of the analysis of the information systems literature - the real artefacts - with the perceptions and intentional acts of information systems researchers using these artefacts.

\section{Providing for perspectives: identifying an appropriate reference ontology}

We have established that journals and their contents are real and have an existence separate from readers and authors, and Ingarden has written extensively on the nature of written works of the type in which we are interested. We note that many see information systems as being a diverse community with many different perspectives and disagreements about categorisation. An appropriate comprehensive reference ontology that is consistent with these traits is needed to provide a framework within which the results of the study of information systems research literature can be presented. 
A number of ontologies have been used to provide an understanding of information systems. A frequently used ontology is that of Mario Bunge (1977; 1979). Bunge's ontology is exact and well developed. It is characterised by an approach that considers the real world as known to science and proceeds in a clear and systematic way. Wand and Weber, alone and with others (e.g. Wand, 1996), have used this ontology in many studies of modelling information systems. However, it has been noted that Bunge's ontology is 'oriented towards the physical world and therefore does not provide for human perceptions and social context' (Wand 1996). Indeed, Bunge's ontology can be categorised as one consistent with the philosophical stream of naturalism wherein it is held that the best methods of inquiry in the social sciences or philosophy are ... those of the natural sciences'. Naturalism is ontologically supported by natural science in that it insists that natural science be used 'in recognising what is real' (Kim and Sosa, 1995) and that 'our ontology is constrained by the result that all physical bodies are composed entirely of particles' (Kim and Sosa, 1995). This position is also methodologically difficult to defend because 'intentional states ... are said to be attributable to individuals only relative to an observer [which is] inconsistent with the objectivity of the methods of natural science' (Winch, 1958, quoted in Kim and Sosa, 1995).

We have established that scientific works such as those in information systems report true judgments of people written with intentions revealed in the works and, furthermore, that people who come from diverse perspectives and with different intentional states read articles. Naturalism cannot help us, despite rightly being a philosophy committed to realism (the existence of a world separate from our thinking about it).

Another ontological position that seems to be more amenable to use in the area of human perceptions is common-sense realism. Commonsensism holds that we really know most, if not all, of those things which ordinary people claim to know. I know that there exists at present a living human body, which is my body. I know that the earth has existed for many years past. These are unambiguous expressions, the meaning of which is widely understood. It should be noted that there is also an 'entirely different question of whether we know what it means, in the sense that we are able to give a correct analysis of its meaning' (Moore, 1925). Commonsensism is not concerned with this latter question. The common-sense world is delineated by our beliefs about what happens in mesoscopic reality in most cases and most of the time (Smith, 1995).

This approach does not dismiss the view of the world based on physics. Various proposals have been made to accommodate the world of physics within common-sense realism. Proposed alternatives have included treating the common-sense world as truly autonomous and the world of physics as a cultural artefact. Smith (1995) proposes that there is an overlap between the common-sense world and the world of physics. Paradigm shifts in science impact on our common-sense understanding of the physical world. However, a common-sense ontology does not necessarily need to be rewritten in the wake of paradigm shifts, contrasting with a naturalist ontology such as Bunge's.

Common-sense realism holds that there is only one world towards which natural cognition relates, and that this world exists independently of our cognitive relations to it. It concedes that our natural cognitive experiences are in many cases unable to be verified, but points out that common sense is aware of error in cognitive efforts.

The thesis that there is only one world towards which natural cognition relates must thus be understood as being compatible with the thesis that there are many different ways in which the world can appear to human subjects in different sorts of circumstances (Smith, 1995). 
An ontology based on common-sense realism, which has received some attention in information systems, is that of Roderick Milton Chisholm (1996). His ontology is consistent with the brand of realism followed in this paper through Husserl, Ingarden, and Brentano. The ontology is robust, located in the common-sense realism school of thought, and deals with static and dynamic aspects. Importantly for the work proposed in this paper, Chisholm addresses the question of perception and the intentional point of view. He states:

I assume that our perception of our own states of mind is a source of certainty and that the deliverances of external perception should be treated as innocent, epistemically, unless we have positive reason to call them into question (Chisholm, 1996: pp. 4-5).

Chisholm's ontology is also able to accommodate 'noema' through 'appearances' thus helping to explain how people (and groups of people) can have perspectives on reality that change over time and appear to be not quite what they really are:

Our qualitative experiences - the sensing of appearances - is subjective in being dependent for its existence on the existence of the subject of experience (Chisholm, 1996, p. 113).

To present the full coverage of Chisholm's ontology is beyond the scope of this paper, but his emphasis on the 'primacy of the intentional' (Chisholm, 1996) suggests that his ontology may provide an appropriate framework for analysing the reality of information systems research. It may be possible to use this well-respected philosophical research to build a sensible categorisation scheme for information systems research, but the question remains of how this can be done.

\section{Establishing and empirically validating ontological categories}

Traditionally, ontological studies have been methodologically based on introspection and analysis of world models and abstract theories. Logic has also been used to analyse the ontological commitments in theories (Quine, 1953). Smith and Mark (1999) have reported on an experiment in the use of empirical methods, based on common-sense realism, to test aspects of an ontological theory of geographic objects. Much of the work done in categorisation is based on mathematical set theory. All objects within a set are equally representative members of a set, and it is absolutely definable as to whether an individual item is or is not a member of a set. In looking at geographical objects, this clarity is not evident. Many geographical terms such as pond, lake, sea or ocean more closely resemble ranges on a continuum rather than precisely definable items. Clearly these geographical objects do not satisfy the requirements of set theory. Equally, comparison of published papers in the field of information systems research with some of the categories proposed in schemes covering information systems research reveal a similar vagueness in distinguishing categories. For example, papers on entity relationship modelling might be categorised as systems analysis and design or as database, or might be thought to lie somewhere between the two, depending on the emphasis of the paper. In these cases we are dealing with categories that are the products of human cognition rather than products of mathematical propositions.

Rosch (1978) looked at the ability of people to differentiate between objects and to identify individual objects which fitted in specific categories better than other individuals, and found a great degree of agreement as to which were good and bad examples. Rather than discrete set-based categories, she described categories with a radial structure, with prototypical or central members surrounded by more or less typical members. This 
has similarities with the approach of Franz Brentano to mereology. The common-sense world is complex and is divided in different ways and at different levels. Mereology concerns the basic organising relationships of part to whole, part to part within a single whole, of identity, overlapping and discreteness.

Most of the work done by Rosch and other cognitive scientists is based on studies of entities of tabletop space such as tools, small pets or of abstract items such as colours and diseases. Smith and Mark (1999) were interested in determining whether these approaches could be applied to geographic categories. Their experimental framework consisted of two complementary phases: traditional ontological work (largely deductive, introspective and formal) and research with human subjects (empirical, inductive). The ontological theories were used as the starting points for the design of experimental protocols to test the degree of fit of the ontological theories. This data can then be used to refine the ontological theories to form the basis for further iterations.

\section{Conclusions}

We have established that the information systems research domain is diverse. We have also established that the approach of Roman Ingarden provides a suitable way to discover the nature of information systems literature - the real world artefacts of information systems research. His work plays a significant part in studies of fiction, plays and poetics. So far, to our knowledge, no exemplars of a research methodology applying his approaches to scientific works exist.

Roman Ingarden's philosophy is in harmony with common-sense realism such as that espoused by Roderick Milton Chisholm and Barry Smith. Common-sense realism provides for perspectival views capturing the diversity of the information systems research domain.

It is likely that this project will require the sort of empirical validation undertaken by Smith and Mark (1999). However, the real objects in their research were not based on intentional acts. Consequently, we believe that no method is directly applicable to undertake this empirical validation, and this remains an active line of enquiry.

The results of this investigation are promising in that a number of different studies, using a common philosophical approach, have been found. When these studies are taken together, they point towards a soundly based, novel methodology for developing a categorial scheme that can be applied in a domain characterised by diversity and, more specifically, diversity of intentionality. 